méthodes par rapport à une sélection utilisant des indices de type "BLUP - modèle animal ", nettement plus efficace que les méthodes de sélection sur performances utilisées dans le tableau 2.

\section{Références}

Archibald A.L. et al, 1995. The PiGMaP consortium linkage map of the pig (Sus scrofa). Mammalian Genome, 6, 157-175.

Argente M.J., Santacreu M.A., Climent A., Bolet G., Blasco A., 1997. Divergent selection for uterine capacity in rabbits. J. Anim. Sci., 75, 2350-2354.

Bidanel J.P., Blasco A., Dando P., Gogué J., Lagant H., 1996a. Résultats de quatre générations de sélection pour le taux d'ovulation et la survie prénatale chez des porcs de race Large White. Journées Rech. Porcine en France, 28, 1-8.

Bidanel J.P., Bonneau M., Chardon P., Elsen J.M., Gellin J., Le Roy P., Milan D., Ollivier L., 1996b. Etablissement et utilisation de la carte génétique porcine. INRA Prod. Anim., 9, 299-310.

Bidanel J.P., Milan D., Chevalet C., Woloszyn N., Bourgeois F., Caritez J.C., Gruand J., Le Roy P., Bonneau M., Lefaucheur L., Mourot J., Prunier A., Désautés C., Mormède P., Renard C., Vaiman M., Robic A., Gellin J., Ollivier L., 1998. Détection de locus à effets quantitatifs dans le croisement entre les races porcines Large White et Meishan. Dispositif expérimental et premiers résultats. Journées Rech. Porcine en France, 30, 117-125.

Blasco A., Bidanel J.P., Bolet G., Haley C.S., Santacreu M.A., 1993. The genetics of prenatal survival of pigs and rabbits : a review. Livest. Prod. Sci., 37, 121.

Bradford G.E., 1969. Genetic control of ovulation rate and embryo survival in mice. I - Response to selection. Genetics, 61, 905-921.

Casey D., Rathje T.A., Johnson R.K., 1994. Second thoughts on selection for components of reproduction in swine. In : Proc. 5th World Congress on Genetics Applied to Livestock Production, 17, 315-318.

Christenson R.K., Leymaster K.A., Young L.D., 1987. Justification of unilateral hysterectomy-ovariectomy as a model to evaluate uterine capacity in swine. J. Anim. Sci., 65, 738-744.
Ellegren H., Chowdhary B., Johansson M., Andersson L., 1994. A primary linkage map of the porcine genome reveals a low rate of recombination. Genetics, 137, 1089-1100.

Jensen E.L., Smith C., Baker L.N., Cox D.F., 1968. Quantitative studies on blood group and serum protein systems in pigs. II. - Effects on production and reproduction. J. Anim. Sci., 27, 856-862.

Johnson R.K., Zimmerman D.R., Kittok R.J., 1984. Selection for components of reproduction in swine. Livest. Prod. Sci., 11, 541-558.

Kirby Y.K., Nielsen M.K., 1993. Alternative methods of selection for litter size in mice : III. Response to 21 generations of selection. J. Anim. Sci., 71, 571-578.

Lamberson W.R., Johnson R.K., Zimmerman D.R., Long T.E., 1991. Direct responses to selection for increased litter size decreased age at puberty, or random selection following selection for ovulation rate in swine. J. Anim. Sci., 69, 3129-3143.

Ollivier L., Messer L.A., Rothschild M.F., Legault C., 1997. The use of selection experiments for detecting quantitative trait loci. Genetical Research, 69, 227232.

Pérez-Enciso M., Bidanel J.P., Baquedano I., Noguera J.L., 1996. A comparison of alternative genetic models for litter size in pigs. Anim. Sci., 63, 255-264.

Rathje T.A., Rohrer G.A., Johnson R.K., 1997. Evidence for quantitative trait loci affecting ovulation rate in pigs. J. Anim. Sci., 75, 1486-1494.

Robison O.W., Lubritz D., Johnson B., 1994. Realized heritability estimates in boars divergently selected for testosterone levels. J. Anim. Breed. Genet., 111, $35-42$.

Rohrer G.A., Alexander L.J., Keele J.W., Smith T.P.L., Beattie C.W., 1994. A microsatellite linkage map of the porcine genome. Genetics, 136, 231-245.

Rohrer G.A., Alexander L.J., Hu Z., Smith T.P.L., Keele J.W., Beattie C.W., 1996. A comprehensive map of the porcine genome. Genome Res., 6, 371391.

Rothschild M.F., Jacobson C., Vaske D.A., Tuggle C., Wang L., Short T., Eckardt G., Sasaki S., Vincent A., McLaren D.G., Souwthwood O., van der Steen H. Mileham A., Plastow, G., 1996. The Estrogen Receptor locus is associated with a major gene influencing litter size in pigs. Proc. Nat. Acad. Sci. (USA), 93, 201-205.

Smith C., 1967. Improvement of metric traits through specific genetic loci. Anim. Prod., 9, 349-358.

\title{
Contrôle du taux d'ovulation chez la truie : l'apport des modèles hyperprolifiques
}

Parmi les nombreuses composantes de la productivité numérique (nombre de porcelets sevrés par truie et par an), la prolificité, ou taille de la portée à la naissance, est devenue un critère prioritaire de sélection. La prolificité dépend de deux facteurs principaux : d'une part le nombre d'ovulations (qui en fixe la limite supérieure) et d'autre part la mortalité embryonnaire et fœtale. Une prolificité élevée peut donc être obtenue via une augmentation du nombre d'ovulations sans réduction de la mortalité embryonnaire (cas de la

\section{M.A. DRIANCOURT, F. MARTINAT-BOTTÉ, M. TERQUI}

INRA Physiologie de la reproduction des mammifères domestiques, 37380 Nouzilly 
lignée hyperprolifique Large White (LWh) : Bolet et al 1986), ou via une réduction de la mortalité embryonnaire sans altération du nombre d'ovulations (cas des truies chinoises: Bazer et al 1988a et b).

Les truies hyperprolifiques constituent un modèle d'analyse original des mécanismes et facteurs de contrôle du nombre d'ovulations. Parallèlement au développement du modèle hyperprolifique en France (Legault et al 1981, Bolet et al 1986), une lignée sélectionnée sur le taux d'ovulation a été créée aux USA (Cunningham et al 1979) (Nebraska selection line). Les caractéristiques de fonctionnement de l'axe hypothalamus-hypophyse-ovaire de ces 2 lignées sont détaillées dans ce texte et comparées chaque fois que cela est possible.

\section{1 / Prolificité et taux d'ovulation dans les 2 modèles}

Lors de la caractérisation initiale des truies hyperprolifiques (LWh) (Bolet et al 1986), celles-ci (truies multipares après 3 portées) présentaient environ 5 ovulations supplémentaires par rapport à la lignée LW témoin. La taille de la portée était accrue d'un porcelet environ, ceci en raison d'une mortalité embryonnaire accrue (LWh : $41 \%$ vs LW : 26 $\%)$.

Ces résultats ont été confirmés dans une étude réalisée à l'INRA de Nouzilly pendant la période 1990-1993 sur 15 truies LWh et 15 truies LW, de poids identique (LWh: $223 \pm 2,4$ vs $\mathrm{LW}: 219 \pm 3,1 \mathrm{~kg}$ ) et de parité identique (LWh : 5,3 $\pm 0,3$ vs LW : 5,7 $\pm 0,5$ portées). Les distributions des nombres d'ovulations chez ces femelles sont présentées sur la figure 1a. Le nombre moyen d'ovulations est significativement $(\mathrm{P}<0,01)$ augmenté chez les LWh $(21,8 \pm 1,3)$ par rapport aux LW $(16,1 \pm 0,7)$.
Les médianes de la fréquence du nombre d'ovulations se situent entre 15 et 18 ovulations chez les LW et entre 20 et 25 chez les LWh. Dans la population hyperprolifique, 3 truies avec des taux d'ovulation exceptionnels ont été identifiées $(26,27$ et 33 ovulations). Cette supériorité ovulatoire des truies hyperprolifiques est déjà présente chez de jeunes truies nullipares (figure 1b). Chez celles-ci, un accroissement (+ 4 environ) du nombre d'ovulations et la présence d'individus exceptionnellement ovulants (jusqu'à 52 ovulations) sont également détectés.

Notons enfin que, dans la population " Nebraska selection line ", après 9 générations de sélection, une supériorité de cette lignée de 3,7 corps jaunes par rapport à la lignée témoin est observée (Cunningham et al 1979)

\section{2 / Caractérisation de la fonction ovarienne chez les Large White hyperprolifiques}

$\mathrm{Au}$ niveau ovarien, deux types de stratégies peuvent générer un nombre d'ovulations plus élevé : soit une folliculogenèse quantitativement plus abondante quelles que soient les classes de taille considérées (cas de la brebis Romanov), soit une altération de la cinétique de développement et de maturation des follicules pendant la phase folliculaire (intervalle régression du corps jaune-ovulation) (cas des brebis Booroola et Finnoises). Ces deux possibilités ont été testées chez la truie hyperprolifique (Driancourt et Terqui 1996).

Dans une première étude, l'ensemble des follicules à antrum ( $>0,2 \mathrm{~mm}$ de diamètre) a été comparé dans les deux ovaires prélevés au stade préovulatoire chez 6 truies LWh et 6 truies LW. Les paramètres mesurés comprenaient les nombres de follicules pour des

Figure 1. Distribution des taux d'ovulation chez:

(a) une petite population ( $n=15$ par génotype) de truies Large White (LW) et hyperprolifiques (LWh) multipares (b) une population importante de LW et LWh nullipares ( $n=156$ et 134 respectivement).
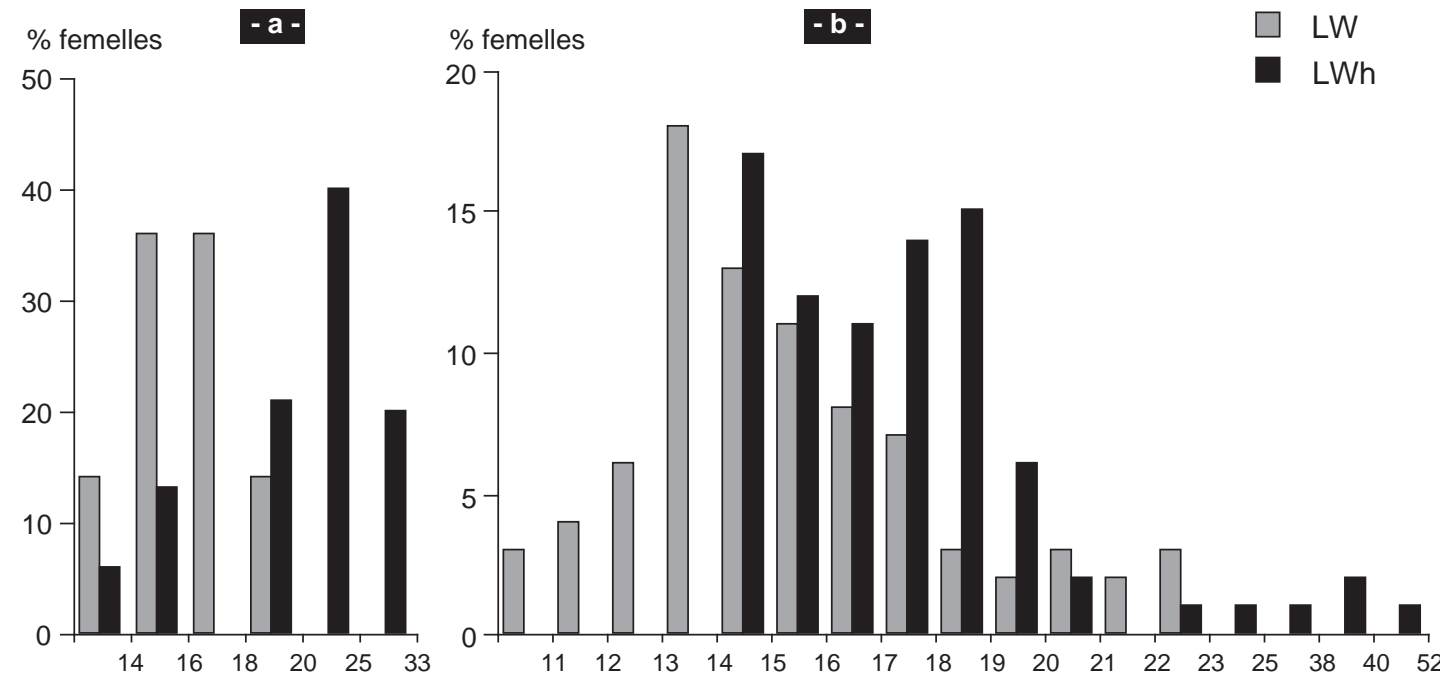
classes de taille déterminées, leur qualité (atrétique ou non) et les caractéristiques des plus gros follicules (taille, nombre de cellules de granulosa....). Les résultats concernant les nombres de follicules (tableau 1), ne montrent aucune différence entre les nombres de follicules totaux dans l'ovaire, leur atrésie et le nombre de follicules (sains ou atrétiques) dans des classes de tailles pré-définies. Il est donc clair que l'hyperprolificité des truies LWh n'est pas liée à une folliculogenèse plus abondante. Cette étude a également mis en évidence la similitude de taille des follicules préovulatoires et du nombre de cellules de granulosa (LWh : 2,9 $\pm 0,2 \times 10^{6}$ vs LW : $2,7 \pm$ $\left.0,1 \times 10^{6}\right)$ dans les 2 lignées.

Dans une seconde étude, la cinétique de croissance des follicules ovulatoires a été étudiée dans chaque lignée par abattage de 5 truies de chaque génotype à chacun des stades suivants : J1, J3 et J5 après la fin d'un traitement de synchronisation au Regumate. Les résultats rapportés dans le tableau 2 mettent en évidence une cinétique de croissance des follicules ovulatoires originale chez les LWh. En effet, si à J3 dans la lignée LW, le nombre de gros follicules (5 à $7 \mathrm{~mm}$ ) est égal au nombre d'ovulations moyen de cette lignée, il ne dépasse pas $17 \mathrm{chez}$ les LWh dont le taux d'ovulation moyen est 22. Cing futur follicules ovulatoires sont encore de taille réduite (entre 3,5 et $5 \mathrm{~mm}$ ) à $\mathrm{J} 3 \mathrm{chez}$ les LWh. A J5, tous les futurs follicules préovulatoires sont de taille supérieure à $5 \mathrm{~mm}$ mais une plus forte proportion d'entre eux est supérieure à $7 \mathrm{~mm}$ chez les LW par rapport aux LWh (75 vs 25 $\%)$. La cinétique de croissance des follicules ovulatoires est donc altérée chez les LWh, du fait d'une période d'apparition des follicules

Tableau 1. Nombre de follicules par classe de taille (classe $1: 0,19$ à $0,36 \mathrm{~mm}$, classe $2: 0,37$ à $0,62 \mathrm{~mm}$, classe $3: 0,63$ à $1,12 \mathrm{~mm}$, classe 4 : 1,13 à $2 \mathrm{~mm}$, classe $5: 2$ à $3,56 \mathrm{~mm}$, classe 6 : $>3,57 \mathrm{~mm}$ ) dans des ovaires de truies hyperprolifiques ou Large White obtenus au stade préovulatoire.

\begin{tabular}{|l|c|c|}
\hline & $\begin{array}{c}\text { Hyper- } \\
\text { prolifique } \\
(\mathrm{n}=6)\end{array}$ & $\begin{array}{c}\text { Large } \\
\text { White } \\
(\mathrm{n}=6)\end{array}$ \\
\hline $\begin{array}{l}\text { Nombre total de follicules } \\
\text { à antrum }\end{array}$ & $646 \pm 105$ & $456 \pm 38$ \\
\% d'atrésie & $62,0 \pm 2,7$ & $66,7 \pm 3,4$ \\
Follicules sains dans & & \\
- classe 1 & $25,1 \pm 8,4$ & $10,1 \pm 3,6$ \\
- classe 2 & $231,3 \pm 35,0$ & $158,3 \pm 23,0$ \\
- classe 3 & $121,0 \pm 31,0$ & $106,0 \pm 12,0$ \\
- classe 4 & $5,5 \pm 3,0$ & $12,0 \pm 6,0$ \\
- classe 5 & 0 & 0 \\
- classe 6 & $23,1 \pm 2,4$ & $18,8 \pm 0,9$ \\
Follicules atrétiques dans & & \\
- classe 2 & $72,0 \pm 13,0$ & $39,0 \pm 10,0$ \\
- classe 3 & $142,0 \pm 37,0$ & $79,0 \pm 6,5$ \\
- classe 4 & $25,0 \pm 7,0$ & $25,0 \pm 1,0$ \\
- classe 5 & $3,3 \pm 1,8$ & $3,7 \pm 1,7$ \\
\hline
\end{tabular}

Aucune différence n'est significative.
Tableau 2. Changements dans les nombres de follicules petits ( 1 à $3,5 \mathrm{~mm}$ ), moyens (3,6 à $5 \mathrm{~mm})$, gros $(5,1$ à $7 \mathrm{~mm}$ ) et très gros (plus de 7,1 mm) chez les truies Large White et hyperprolifiques au cours de la phase folliculaire (Driancourt et Terqui 1996).

\begin{tabular}{|l|c|c|}
\hline & $\begin{array}{c}\text { Hyper- } \\
\text { prolifique }\end{array}$ & $\begin{array}{c}\text { Large } \\
\text { White }\end{array}$ \\
\hline J1 & & \\
Total & $74,0 \pm 4,1$ & $67,6 \pm 6,8$ \\
Petits & $53,8 \pm 4,4$ & $39,8 \pm 8,3$ \\
Moyens & $17,8 \pm 2,8$ & $21,0 \pm 2,8$ \\
Gros & $2,4 \pm 1,2$ & $6,4 \pm 2,1$ \\
Très Gros & 0 & 0 \\
J3 & & \\
Total & $50,2 \pm 5,2$ & $49,2 \pm 5,5$ \\
Petits & $21,0 \pm 5,0$ & $20,0 \pm 5,0$ \\
Moyens & $12,0 \pm 2,2$ & $11,4 \pm 2,1$ \\
Gros & $17,2 \pm 2,1$ & $18,0 \pm 1,6$ \\
Très Gros & 0 & $0,2 \pm 0,2$ \\
J5 & & \\
Total & $30,6 \pm 1,6$ & $29,8 \pm 2,8$ \\
Petits & $8,0 \pm 2,2$ & $4,8 \pm 1,0$ \\
Moyens & $1,6 \pm 0,5$ & $4,2 \pm 1,1$ \\
Gros & $18,2 \pm 2,0$ & $4,8 \pm 1,0$ \\
Très Gros & $6,0 \pm 1,6$ & $12,8 \pm 1,3$ \\
\hline
\end{tabular}

ovulatoires plus étalée dans le temps (de J1 à J5 chez les LWh, seulement de J1 à J3 chez les LW).

Dans la lignée "Nebraska selection line", des conclusions très proches ont été obtenues : pas de différences dans la population folliculaire, cinétique de développement des follicules ovulatoires modifiée (Kelly et al 1988a, Vatzias et al 1991).

Dans un dernier volet de cette étude, la maturation (mesurée par l'aptitude des follicules à produire de l'œstradiol et de la testostérone in vitro) des follicules obtenus à J1, J3 et J5 dans les 2 lignées a été comparée (Driancourt et Terqui 1996). Les résultats, présentés au tableau 3 , concernent 70 et 78 follicules de LWh et LW à J1, 94 et 94 follicules de LWh et LW à J3 ainsi que 83 et 88 follicules de LWh et LW à J5, jugés sains au vu de la production de stéroïdes. Pour la taille folliculaire, un effet stade de la phase folliculaire $(\mathrm{P}<0,01)$ et un effet génotype $(\mathrm{P}<0,01)$ ont été mis en évidence. A J3 et à J5, les follicules sains sont plus petits $(\mathrm{P}<0,01)$ chez les LWh que chez les LW. En ce qui concerne la production de testostérone, seul un effet stade de la phase folliculaire a été mis en évidence. En revanche, la comparaison des productions d'œstradiol a permis d'identifier un effet stade $(\mathrm{P}<0,01)$, ainsi qu'un effet génotype $(\mathrm{P}<0,05)$ car, à $\mathrm{J} 3$ et à J5, la production d'œstradiol des follicules de LWh est supérieure à celle des follicules de LW. La supériorité de la production d'œstradiol chez les LWh, alors que la quantité de précurseurs (testostérone) est identique dans les 2 lignées, suggère que l'aromatase (enzyme 
Tableau 3. Moyennes des moindres carrés de la taille et de la stéroïdogenèse folliculaire pour les follicules de truies Large White et hyperprolifiques à 3 stades de la phase folliculaire (Driancourt et Terqui 1996).

\begin{tabular}{|l|c|c|}
\hline & $\begin{array}{c}\text { Hyper- } \\
\text { prolifique }\end{array}$ & $\begin{array}{c}\text { Large } \\
\text { White }\end{array}$ \\
\hline Taille (mm) & $4,71 \pm 0,103$ & $4,89 \pm 0,065^{\text {a }}$ \\
J1 & $5,58 \pm 0,059^{\text {a }}$ & $5,88 \pm 0,058^{\text {c }}$ \\
J3 & $6,79 \pm 0,056^{\text {a }}$ & $7,54 \pm 0,06^{\text {c }}$ \\
J5 & & \\
Testostérone (ng/ml) & $1,58 \pm 2,98$ & $1,35 \pm 1,88$ \\
J1 & $22,10 \pm 2,94$ & $18,00 \pm 1,62$ \\
J3 & $48,90 \pm 1,60$ & $66,20 \pm 1,70$ \\
J5 & & \\
Oestradiol (ng/ml) & $2,0 \pm 1,2$ & $1,1 \pm 0,8$ \\
J1 & $12,2 \pm 1,2^{\text {a }}$ & $9,9 \pm 0,7$ b \\
J3 & $33,4 \pm 0,6^{\text {a }}$ & $22,1 \pm 0,7^{\text {c }}$ \\
J5 &
\end{tabular}

Les différences entre lignées sont significatives à $\mathrm{P} \square<\square 0,1$ (a vs b) ou $\mathrm{P} \square \square 0,01$ (a vs c).

convertissant les androgènes en œstradiol) est plus abondante dans les follicules de LWh lorsqu'ils atteignent une taille ovulatoire. Une telle conclusion est manifeste à l'examen de la figure 2 où, quelles que soient les concentrations de testostérone disponibles, les follicules de Lwh produisent plus d'œstradiol. En conclusion, en période préovulatoire, les follicules de truies LWh sont caractérisés par une concentration d'aromatase (dans la granulosa et/ou la thèque) augmentée et par un nombre de cellules de granulosa identique à celui des follicules de LW témoin. Cette situation est très différente de la situation observée chez les brebis porteuses du gène Booroola pour lesquelles la taille et le nombre de cellules de granulosa sont fortement réduits (d'au moins

Figure 2. Régressions reliant production d'œstradiol et production de testostérone chez des truies Large White (LW) et hyprerprolifiques (LWh).

Production d'oestradiol ( $\mathrm{ng} / \mathrm{ml} / \mathrm{h})$

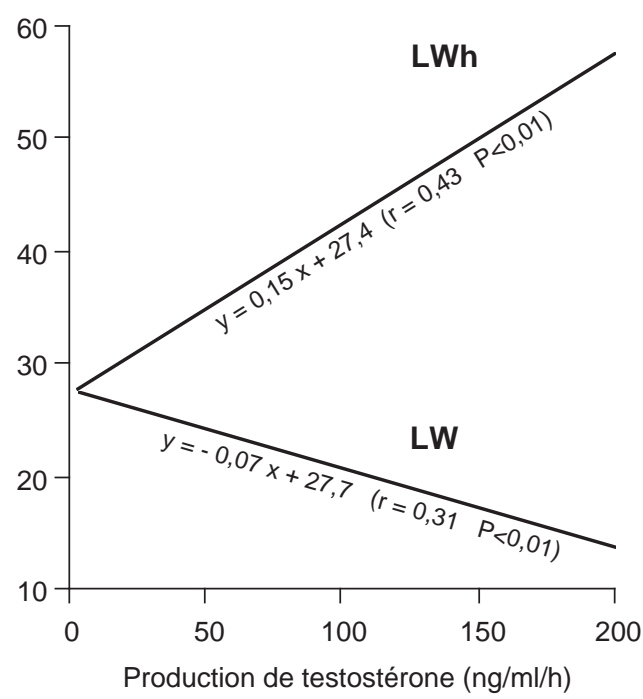

$50 \%$ ) chez les porteuses et l'aromatase n'est pas affectée (Montgomery et al 1992).

\section{3 / Pourquoi les truies LWh ovulent-elles plus que les LW}

Chez la truie, la croissance folliculaire à partir d'environ $2 \mathrm{~mm}$ est totalement dépendante des hormones gonadotropes (Kraeling et al 1990, Driancourt et al 1995). De plus, les expériences d'ablation et/ou de supplémentation ont permis d'identifier une phase FSH dépendante ( 2 à $4 \mathrm{~mm}$ ) suivie d'une phase $\mathrm{LH}$ dépendante (4 à $8 \mathrm{~mm}$ ) au cours de la croissance vers l'ovulation des follicules (Guthrie et al 1990, Driancourt et al 1995). L'importante de la sécrétion de LH pour la fin de la croissance ovulatoire est encore soulignée par les observations montrant (1) une réponse supérieure à $\mathrm{LH}$ (par rapport à FSH) mesurée par la production d'AMP cyclique par les cellules de la granulosa (Guthrie et al 1993) et (2) l'absence d'ARNm codant pour le récepteur à FSH dans la granulosa des follicules de grande taille $(=7 \mathrm{~mm}$ ) (Yuan et al 1996).

La mesure des niveaux de FSH et de ceux de LH (incluant particulièrement l'analyse de la pulsatilité de la $\mathrm{LH}$ ) constitue donc une étape incontournable de la réflexion sur le contrôle du nombre d'ovulation chez les porcins. Elle n'a jamais été réalisée par une comparaison LWh vs LW. Cependant, cette question a été partiellement abordée chez les truies «Nebraska sélection line » (Kelly et al 1988b). Les niveaux moyens de FSH et de LH dans les 48 heures qui précèdent le pic de LH sont identiques chez les truies de la lignée sélectionnée et chez les témoins. La pulsatilité de la $\mathrm{LH}$ pendant cette période, ainsi que les niveaux de FSH et LH en début de phase folliculaire n'ont pas été mesurés. Une caractérisation des profils d'hormones gonadotropes dans les lignées LWh et LW doit donc être réalisée. Si une supériorité des niveaux de FSH et/ou de LH est observée chez les LWh, la vérification d'un lien de cause à effet entre ces niveaux et l'augmentation du nombre d'ovulations devra être faite en développant un modèle dans lequel la croissance folliculaire est bloquée par un antagoniste de LHRH et ré-induite par des niveaux contrôlés de FSH et LH.

Cette hypothèse "gonadotrope " n'est cependant pas la seule susceptible d'expliquer une élévation du nombre d'ovulations. Ainsi chez les brebis Finnoises d'une lignée haute sélectionnée sur un taux d'ovulation élevé (4 ovulations au lieu de 2,5 chez la lignée témoin), les concentrations de FSH sont identiques à celles mesurées dans la lignée témoin (Adams et al 1988).

Trois types de mécanismes (au moins !) sont susceptibles d'expliquer une meilleure efficacité au niveau ovarien du signal gonadotrope. 


\section{L'amplification par l'œstradiol de la réponse ovarienne à LH et FSH}

Chez la ratte, en effet, Richards (1980) a montré que l'œstradiol potentialisait l'action de FSH sur l'induction des récepteurs à $\mathrm{LH}$ sur la granulosa. De plus, chez la ratte, l'œstradiol exerce un effet protecteur sur l'atrésie induite par l'ablation des hormones gonadotropes après hypophysectomie (Billig et al 1993). Les résultats obtenus chez la truie par Veldhuis et al (1982) suggèrent que l'œstradiol pourrait avoir un effet identique. La production d'œstradiol plus élevée des follicules de truies LWh dès J3 pourrait alors avoir un effet favorisant la différenciation des récepteurs à LH des follicules les plus petits dans le groupe de follicules ovulatoires.

\section{Des interactions paracrines (entre tissus constitutifs du follicule) ou autocrines (intra-tissu) altérées}

Les candidats (protéines, facteurs de croissance) et les altérations possibles sont extrêmement nombreux. Seuls quelques exemples confortant la validité de cette hypothèse sont présentés ci-dessous :

- l'ovocyte est capable de moduler la production d'œstradiol induite par FSH chez la souris (Vanderhyden et al 1993). Notons également que la croissance et la maturation ovocytaires sont affectées par la présence du gène FecB Booroola (Cognié et al 1998). La démonstration d'une telle régulation reste néanmoins à effectuer chez les porcins ;

- un tissu (la granulosa) produit une protéine ou un facteur de croissance qui amplifie l'action de FSH sur ce même tissu. Par exemple, sur des cellules de granulosa de primates, Hillier et Miro (1992) ont montré que l'activine (dimère $\beta$ - $\beta$ d'une sous-unité de l'inhibine) potentialisait l'action inductrice de FSH sur l'aromatase, et qu'au cours de la croissance vers l'ovulation, les follicules perdraient progressivement leur aptitude à produire de l'activine de façon synchrone de l'acquisition de l'aptitude à produire de l'inhibine. Des informations sur la famille inhibine/activine dans l'ovaire de truie ne sont pas disponibles, mais une altération de la dynamique de production de l'inhibine et de l'activine chez les LWh par arpport aux LW est un des mécanismes pouvant expliquer l'altération de la cinétique du développement des follicules ovulatoires observée chez les LWh.

Un autre exemple de régulation autocrine, caractérisé au moins partiellement chez les porcins, concerne la famille des "Insulin like Growth factors " (IGF1 et IGF2) et de leurs protéines de liaison (IGF BP). L'ovaire de truie, et particulièrement la granulosa des follicules sains, est capable de synthétiser les ARNm des IGF1 et IGF2 ainsi que ceux d'IGF BP2 et BP4 (Zhou et al 1996). De plus, l'IGF1 et au moins 5 protéines de liaison sont présents dans le fluide folliculaire porcin (Howard et Ford 1992). Au cours de la croissance ovulatoire, les quantités d'IGF BP présentes dans le fluide folliculaire diminuent
(Howard et Ford 1992), augmentant ainsi la biodisponibilité de l'IGF1. Comme l'IGF1 exerce un effet amplificateur de l'action de FSH sur la granulosa et de LH sur la thèque, un tel mécanisme peut moduler localement l'action des hormones gonadotropes. Les profils de protéines de liaison des IGF chez les LWh n'ont, pour l'instant, pas été comparés à ceux des LW.

\section{Une meilleure efficacité de la distribution à l'ovaire des hormones gonadotropes}

L'approvisionnement folliculaire en nutriments et hormones dépend essentiellement de deux facteurs : d'une part la microvascularisation de chaque follicule, un paramètre contrôlé par deux facteurs de croissance (FGF, Fibroblast Growth Factor, VEGF Vascular Endothelial Growth Factor) ; d'autre part, l'existence de systèmes locaux de recirculation au niveau du pédicule de l'ovaire (Kzymowski et al 1982).

Une vascularisation plus efficace des follicules ou de l'ovaire chez les truies LWh pourrait alors améliorer l'utilisation ovarienne des hormones gonadotropes.

\section{Conclusion}

Le bilan des données accumulées sur les modèles génétiques de l'hyperovulation chez les porcins montre qu'il existe, à l'heure actuelle, beaucoup plus d'hypothèses que de certitudes. Une conclusion claire émerge cependant : dans le modèle hyperprolifique, des altérations dans la sensibilité de l'axe hypothalamo-hypophysaire au feed back négatif des stéroïdes et de l'œstradiol doivent exister pour contrebalancer la production accrue d'œstradiol (résultat de la synergie entre un nombre de follicules augmenté et une production d'œstradiol par follicule également augmentée). La fonction de l'ensemble de l'axe hypothalamus-hypophyse-ovaire est donc sans doute modifiée chez les LWh.

La caractérisation fine des mécanismes (endocriniens ou ovariens) produisant l'augmentation du nombre d'ovulations chez les LWh devra être réalisée. Cependant, pour que cette caractérisation soit fructueuse, une population témoin doit être identifiée. Faut-il alors comparer les femelles à taux d'ovulation exceptionnel (plus de 25 ovulations) aux autres? Faut-il pratiquer une sélection divergente chez les LWh?

\section{Références}

Adams T.E., Quirke J.F., Hanrahan J.P., Adams B.M., Watson J.G., 1988. Gonadotropin secretion during the periovulatory period in Galway and Finn ewes selected for high ovulation rate. J. Reprod. Fert., 83, 575-583. 
Bazer F.W., Thatcher W.W., Martinat-Botté F., Terqui M., 1988a. Conceptus development in Large White and prolific Chinese Meishan pigs. J. Reprod. Fert., 84, 37-44.

Bazer F.W., Thatcher W.W., Martinat-Botté F., Terqui M., 1988b. Sexual maturation and morphological development of the reproductive tract in Large White and prolific Chinese Meishan pigs. J. Reprod. Fert., 83, 723-730.

Billig H., Furuta I., Hsueh A.J.W., 1993. Estrogens inhibit and androgen enhance ovarian granulosa cell apoptosis. Endocrinology, 133, 2204-2212.

Bolet G., Martinat-Botté F., Locatelli A., Gruard J., Terqui M., Berthelot F., 1986. Components of prolificacy in hyperprolific Large White sows compared with the Meishan and Large White breeds. Genet. Sel. Evol., 18, 333-342

Cognié Y., Benoit F., Poulin N., Khatir H., Driancourt M.A., 1998. Oocyte function in sheep ; effect of follicle size and of the FecB Booroola gene. J. Reprod. Fert., 112, 379-386.

Cunningham P.J., England M.E., Young L.D., Zimmerman D.R., 1979. Selection for ovulation rate in swine ; correlated response in litter size and weight. J. Anim. Sci., 48, 509-517.

Driancourt M.A., Terqui M., 1996. Follicular growth and maturation in hyperprolific and Large White sows. J. Anim. Sci., 74, 2231-2238.

Driancourt M.A., Locatelli A., Prunier A., 1995 Effects of gonadotrophin deprivation on follicular growth in gilts. Reprod. Nutr. Dev., 35, 663-673.

Guthrie H.D., Bolt D.J., Cooper B.S., 1990. Effects of gonadotropin treatment on ovarian follicle growth and granulosa cell aromatase activity in prepuberal gilts. J. Anim. Sci., 68, 3719-3726.

Guthrie H.D., Bolt D.J., Cooper B.S., 1993. Changes in follicular ostradiol $17 \beta$, progesterone and inhibin immunoactivity in healthy and atretic follicles during preovulatory maturation in the pig. Dom Anim. Endocr., 10, 127-140.

Hillier S.G., Miro F., 1992. Relative effects of activin and inhibin on steroid hormone synthesis in primate oranulosa cells. J. Clin. Endocr. Metab., 75, 1556 1561.

Howard H.J., Ford J.J., 1992. Relationship among concentrations of steroids, inhibin, IGF1 and IGF binding proteins during follicular development in weaned sows. Biol. Reprod., 47, 193-201.

Kelly C.R., Kopf J.D., Zimmerman D.R., 1988a. Characterization of antral follicle populations during the estrous cycle in pigs selected for ovulation rate. J. Anim. Sci., 66, 1230-1235.
Kelly C.R., Socha T.E., Zimmerman D.R., 1988b. Characterization of gonadotropic and ovarian steroid hormones during the periovulatory period in high ovulating select and control line gilts. J. Anim. Sci. $66,1462-1474$.

Kraeling R.R., Kesner J.S., Estienne M.J., Estienne C.E., Barb C.R., Rampacek G.B., 1990. Follicle growth in hypophysial stalk transected pigs given pulsatile GnRH and PMSG. Dom. Anim. Endocr., 7, 395-402.

Krzymoski T., Kotwica J., Stefanczyk S., Czarnocki J., Debek J., 1982. A sub-ovarian exchange mechanism for the countercurrent transfer of ovarian steroid hormones in the pig. J. Reprod. Fert., 65, 457465 .

Legault C., Gruand J., Bolet G., 1981. Résultats de l'utilisation en race pure et en croisement d'une lignée dite "hyperprolifique ». Journées de la Recherche Porcine en France, 13, 261-267. ITP, Paris.

Montgomery G.W., McNatty K.P., Davis G.H., 1992 Physiology and molecular genetics of mutations that increase ovulation rate in sheep. Endocrine Reviews, $13,309-328$.

Richards J.S., 1980. Maturation of ovarian follicles actions and interactions of pituitary and ovarian hormones on follicular cell differenciation. Phys. Rev., 60, 51-87.

Vanderhyden B.C., Cohen J.N., Morley P., 1993. Mouse oocytes regulate granulosa cell steroidogenesis. Endocrinology, 133, 423-427.

Vatzias G., Knox R., Naber C., Zimmerman D.R., 1991. Further characterization of changes in ovarian follicle populations during the follicular phase as affected by genetic selection for high ovulation rate in gilts. J. Anim. Sci., 69 (suppl. 1), 432 (Abst).

Veldhuis J.D., Klase P.A., Strauss J.F., Hammond J.M., 1982. The role of estradiol as a biological amplifier of the actions of follicle stimulating hormone : in vitro studies in swine granulosa cells. Endocrinology, 111, 144-151.

Yuan W., Lucy M.C., Smith M.F., 1996. Messenger Ribonucleic acid for insulin like growth factors 1 and 2, IGFBP2, gonadotrophin receptors and steroidogenic enzymes in porcine follicles. Biol. Reprod., 55 , 1045-1054.

Zhou J., Adesanya O.O., Vatzias G., Hammond J.M., Bondy C.A., 1996. Selective expression of Insulinlike Growth factor system components during porcine ovary follicular selection. Endocrinology, 137 4893-4901.
M. TERQUI, F. MARTINAT-BOTTÉ

INRA Station de Physiologie de la Reproduction des Mammifères Domestiques, 37380 Nouzilly

\section{Mortalité embryonnaire et prolificité chez la truie}

Une ovulation n'est pas obligatoirement associée à la naissance d'un jeune. En fait, celle-ci va dépendre de la survie embryonnaire (nombre d'embryons vivants à 30 jours de gestation par rapport au nombre d'ovulations) et de la survie fotale (nombre de foetus ou de porcelets par rapport au nombre d'embryons). La survie embryonnaire est l'élément principal qui conditionne la taille de la portée et elle varie de façon importante. Ainsi, elle est de 66,68 et $93 \%$ respectivement chez les truies Large White, Large White hyperproli- 